\title{
Evaluation of Haemagglutinin Content by RP-HPLC to Generate Pandemic Influenza Vaccine
}

\author{
Hyunkyung Kang ${ }^{1 \dagger}$, Hang Sik Roh ${ }^{1 \dagger}$, Hyemin Song ${ }^{1}$, Kwangmoon Lee $^{1}$, Seung-Tae Chung ${ }^{1}$, \\ Sang-ja Ban ${ }^{1}$, In Pil Mo ${ }^{2}$, Beum-Soo An ${ }^{3}$ and Chi-Young Ahn' \\ ${ }^{1}$ Biologics Research Division, National Institute of Food and Drug Safety and Evaluation, Chungwon, Korea \\ ${ }^{2}$ College of Veterinary Medicine, Chungbuk National University, Cheongju, Korea \\ ${ }^{3}$ Department of Biomaterials Science, College of Natural Resources \& Life Science/Life and Industry Convergence Research Institute, \\ Pusan National University, Miryang, Korea
}

(Received April 7, 2016; Revised July 29, 2016; Accepted August 1, 2016)

\begin{abstract}
The potency of influenza vaccine is determined based on its hemagglutinin (HA) content. In general, single radial immunodiffusion (SRID) assay has been utilized as the standard method to measure HA content. However, preparation of reagents for SRID such as antigen and antibody takes approximately 2 3 months, which causes delays in the development of influenza vaccine. Therefore, quantification of HA content by other alternative methods is required. In this study, we measured HA contents of H1N1 antigen and H1N1 influenza vaccine by reverse phase-high performance liquid chromatography (RP-HPLC) methods. The presence of HA1 and HA2 was investigated by silver staining and Western blot assay. In addition, accuracy and repeatability of HA measurement by RP-HPLC were evaluated. Comparison of HA concentration by SRID and RP-HPLC revealed a precise correlation between the two methods. Our results suggest that RP-HPLC assay can replace SRID in the event of a pandemic flu outbreak for rapid vaccine development.
\end{abstract}

Key words: Influenza, Hemagglutinin, RP- HPLC, Vaccine

\section{INTRODUCTION}

Influenza is an infectious viral disease with seasonal epidemicity and can cause inconvenience in healthy people.

Correspondence to: Chi Young Ahn, Biologics Research Division, National Institute of Food and Drug Safety Evaluation, KFDA, 187 Osongsaengmyeong2-ro, Cheongwon-gun, Chungcheongbuk-do 28159, Korea

E-mail: 72notul@korea.kr

Beum-Soo An, Department of Biomaterials Science, College of Natural Resources and Life Science, Pusan National University, Miryang 50463, Korea

E-mail: anbs@pusan.ac.kr

${ }^{\dagger}$ The first two authors contributed equally to this work.

Abbreviations: HA, Hemagglutinin; SRID, Single radial immunodiffusion; RP-HPLC, Reverse phase-high performance liquid chromatography; NA, Neuraminidase; WHO, World Health Organization; HPLC, High-performance liquid chromatography; DTT, Dithiothreitol; IAA, lodoactamide; TFA, Trifluoroacetic acid; LOD, Limit of detection; LOQ, Limit of quantitation.

This is an Open-Access article distributed under the terms of the Creative Commons Attribution Non-Commercial License (http:// creativecommons.org/licenses/by-nc/3.0) which permits unrestricted non-commercial use, distribution, and reproduction in any medium, provided the original work is properly cited.
Influenza vaccine is the most effective and surest way to prevent influenza. The viral strains contained in influenza vaccines are changed almost every year as the epidemic strains change. In addition to regular recurrence of influenza, epidemics are caused by viral antigenic drift (1). The outermost part of the influenza virus is an envelope structure composed of double lipid layers containing two surface glycoproteins: a rod-shaped hemagglutinin (HA) and a mushroom-shaped neuraminidase (NA). The main antigen of influenza vaccine is derived from HA, and potency of the vaccine is based on HA content. Today, the single radial immunodiffusion technique (SRID) recommended by the European Pharmacopoeia and World Health Organization (WHO) is commonly used to measure the HA content of a vaccine (2). In this assay, a reference homologous HA antigen together with specific antibodies are required for HA calibration. The SRID method is very specific, does not need advanced techniques, and serves as the only method to visually measure the antigen content of a vaccine (3). However, there is a need to develop rapid techniques for assessment of HA content in case urgent vaccine development against an influenza pandemic outbreak. Indeed, supply of international standard antigen from the WHO was delayed in 2009, and Korea experienced a resultant delay of vaccine production and follow-up clinical tests during the 
H1N1 influenza pandemic. As it takes a relatively long time to develop standard antigen and antibody, which are essential for assessment of HA content using the SRID method $(4,5)$, a new and prompt HA content assessment method is needed. Thus, efforts have been made to develop methods for assessing HA content in influenza vaccines using a physicochemical approach, including high-performance liquid chromatography (HPLC) (6-8). In this study, we developed a calibration method measuring HA content from the HA1 peak area (6-8). Using this method, we measured HA content from 22 lots of seasonal influenza monovalent bulk vaccine and compared those values with results from the SRID method.

\section{MATERIALS AND METHODS}

Chemicals. All chemicals were of analytical reagent grade. $\mathrm{NH}_{4} \mathrm{HCO}_{3}$, dithiothreitol (DTT), iodoactamide (IAA), and trifluoroacetic acid (TFA) were obtained from Sigma (St. Louis, MO, USA). Trypsin conjugated to agarose beads was purchased from Pierce (Rockford, IL, USA). Zwittergent was obtained from Calbiochem (Billerica, MA, USA). Acetonitrile was purchased from Burdick and Jackson (Muskegon, MI, USA). Distilled water was deionized on a LabTower $^{\mathrm{TM}}$ EDI50 system (Thermo scientific, Waltham, MA, USA).

Vaccines and the standard materials. The vaccines used in this study were seasonal influenza vaccines containing the A/California/7/2009 (H1N1) strain (H1N1 vaccine, Green Cross Corp., Yongin, Korea). The antigens for A/ California/7/2009 (H1N1) (H1N1 antigen, NIBSC code 09/ 294 and 11/202) prepared by NIBSC (London, UK) were used as reference standards for calibration. The standard anti-serum for the SRID test was Influenza Anti-A/California/7/2009 (H1N1) HA serum (H1N1 anti-HA serum, NIBSC code 11/110, Green Cross Corp.).

Standard and sample preparation for RP-HPLC method. The experimental conditions for the RP-HPLC method were based on the protocol presented by Kapteyn et al. $(6,7)$. Standard antigens were reconstructed with $0.1 \mathrm{M}$ $\mathrm{NH}_{4} \mathrm{HCO}_{3}$ and diluted to four different concentrations for calibration. The vaccine was serially diluted in the same manner with standard antigens. Each calibrant $(200 \mu \mathrm{L})$ was kept at room temperature for 10 min after mixing with $1 \%$ zwittergent solution. Agarose beads conjugated with trypsin (24 TAME units) were added to the solution and incubated at $37^{\circ} \mathrm{C}$ for $3 \mathrm{hrs}$ with shaking. After centrifugation, the supernatant was taken and mixed with $25 \mathrm{mM}$ DTT at $90^{\circ} \mathrm{C}$ for $5 \mathrm{~min}$, cooled down to room temperature, alkylated by addition of $50 \mathrm{mM}$ IAA, and then incubated at $37^{\circ} \mathrm{C}$ for $10 \mathrm{~min}$ in the dark. After neutralization of IAA by addition of $25 \mathrm{mM}$ DTT, the assessment process was car- ried out. The H1N1 vaccine was diluted 2 or 3-fold with $0.1 \mathrm{M} \mathrm{NH}_{4} \mathrm{HCO}_{3}$ and pretreated under the same conditions with standard antigen.

RP-HPLC conditions. The HPLC conditions followed those of Kapteyn et al. $(6,7)$. Briefly, the standard antigen and vaccine samples were analyzed on a Waters Alliance 2695 system using a polystyrene POROS R1/10 (2.1 mm $\times$ $100 \mathrm{~mm}$ ) column and Waters 2996 PDA detector. The mobile phase $\mathrm{A}$ was a $5 \%$ acetonitrile and $0.1 \%$ TFA solution, whereas the mobile phase $\mathrm{B}$ was a $0.1 \%$ TFA acetonitrile solution. The flow rate was $0.8 \mathrm{~mL} / \mathrm{min}$, and a UV detector at $214 \mathrm{~nm}$ was used. The gradient elution for solvent $B$ was $10 \%$ as the start, stepped to $20 \%$ for $4 \mathrm{~min}$, retained for $2 \mathrm{~min}$, stepped to $25 \%$ for $1 \mathrm{~min}$, retained for $3 \mathrm{~min}, 30 \%$ for $1 \mathrm{~min}, 35 \%$ for $3 \mathrm{~min}, 100 \%$ for $1 \mathrm{~min}$, retained for $1 \mathrm{~min}$, stepped to $10 \%$ for $1 \mathrm{~min}$, and then retained for $25 \mathrm{~min}$ for re-equilibration. The autosampler for cooling and column heater were kept at $4^{\circ} \mathrm{C}$ and $65^{\circ} \mathrm{C}$, respectively.

Method validation parameters. Linearity and precision were evaluated, and the limit of detection (LOD) and limit of quantitation (LOQ) were measured for validation of the RP-HPLC method. Five different concentrations of $\mathrm{H} 1 \mathrm{~N} 1$ vaccine were prepared and analyzed in order to demonstrate that quantification was not affected by HA concentration. Plots of the HA1 peak area were prepared, and linearity for HA1 concentration was assessed through the coefficient of correlation $\left(\mathrm{R}^{2}\right)$ of the regression line. The LOD was determined by measurement of the signal-tonoise ratio for decreasing amounts injected. The LOD was set as the lowest amount injected, giving $\mathrm{S} / \mathrm{N} \geq 3$. The LOQ was determined as the lowest amount injected, giving $\mathrm{S} /$ $\mathrm{N} \geq 10$. To assess repeatability, replicate injection of H1N1 vaccine was carried out during a single experiment, and the retention time and peak area were measured.

SRID assay. In the SRID assay, HA content was measured from 22 lots of seasonal H1N1 vaccines. SRID was performed based on the method by Wood et al. (9), and the reagents and equipment settings were adjusted for this study. The standard H1N1 antigen and H1N1 vaccine were diluted with water to similar concentrations, incubated with $1 \%$ zwittergent solution at room temperature for $30 \mathrm{~min}$, and subjected to immunodiffusion for at least $18 \mathrm{hrs}$ at room temperature in antibody-loaded agarose gels. Diameters of the precipitation rings of antigen-antibody complexes were measured by a computerized ProtoCOL system.

SDS-PAGE/silver staining and Western blot analysis. Vaccine samples used RP-HPLC analysis and RP-HPLC fractions were subjected to Western blot analysis and SDSPAGE/silver staining. SDS-PAGE NuPAGE ${ }^{\circledR}$ NOVEX BT 
$4 \sim 12 \%$ gels and silver staining kit (SilverXpress ${ }^{\circledR}$, Invitrogen Co., Carlsbad, CA, USA) were used for silver staining. H1N1 anti-HA serum was used for specific detection of HA by Western blotting.

\section{RESULTS}

HA1 peak isolation from RP-HPLC. To identify and separate HAs from standard H1N1 antigens and H1N1 vaccines, various concentrations of trypsin and incubation times were tested, and the separation was confirmed by Western blotting (data not shown). For standard H1N1 antigen, HA was separated into HA1 and HA2 after treatment of antigen with trypsin for $3 \mathrm{hrs}$ at $37^{\circ} \mathrm{C}$. Under the same experimental conditions, both standard H1N1 antigen (peak 2 in Fig. 1A) and H1N1 vaccines (peak 3 in Fig. 1B) showed an HA1 peak at around the 12-min slot. To confirm the isolated HAs from H1N1 antigen and H1N1 vaccine, fractions of the peaks were processed by silver staining (Fig. 2A and 2C) and Western blot assay (Fig. 2B and 2D). Silver staining revealed fractional proteins for peaks 2 and 3 from $\mathrm{H} 1 \mathrm{~N} 1$ antigen and peak 3 from $\mathrm{H} 1 \mathrm{~N} 1$ vaccine with a size of $51 \mathrm{kDa}$, which is the same as the molecular weight of HA1. In Western blotting, the fraction for peak 2 from $\mathrm{H} 1 \mathrm{~N} 1$ antigen and peak 3 from $\mathrm{H} 1 \mathrm{~N} 1$ vaccine specifically reacted with anti-HA serum of H1N1 but not with other peak fractions, suggesting that peak 2 from H1N1 antigen and peak 3 from H1N1 vaccine corresponds to HA1 protein. These results mean that the fractions from the 12-minute slot for both $\mathrm{H} 1 \mathrm{~N} 1$ antigen and $\mathrm{H} 1 \mathrm{~N} 1$ vaccine can be designated as HA1 protein. HA2 signal was also detected in peaks 4 and 5 for H1N1 antigen and H1N1 vaccine, although the signals were relatively weaker than those of HA1.

Method validation. Method validation was also carried out to investigate the validity of the established analysis method. H1N1 antigen and vaccine were processed for RP-HPLC in an HA1 concentration-dependent manner, after which the areas corresponding to the HA1 peak (peak 2 for $\mathrm{H} 1 \mathrm{~N} 1$ antigen and peak 3 for $\mathrm{H} 1 \mathrm{~N} 1$ vaccine) were evaluated (Fig. 3). In the results, favorable linearity was detected in five different concentrations of H1N1 antigen
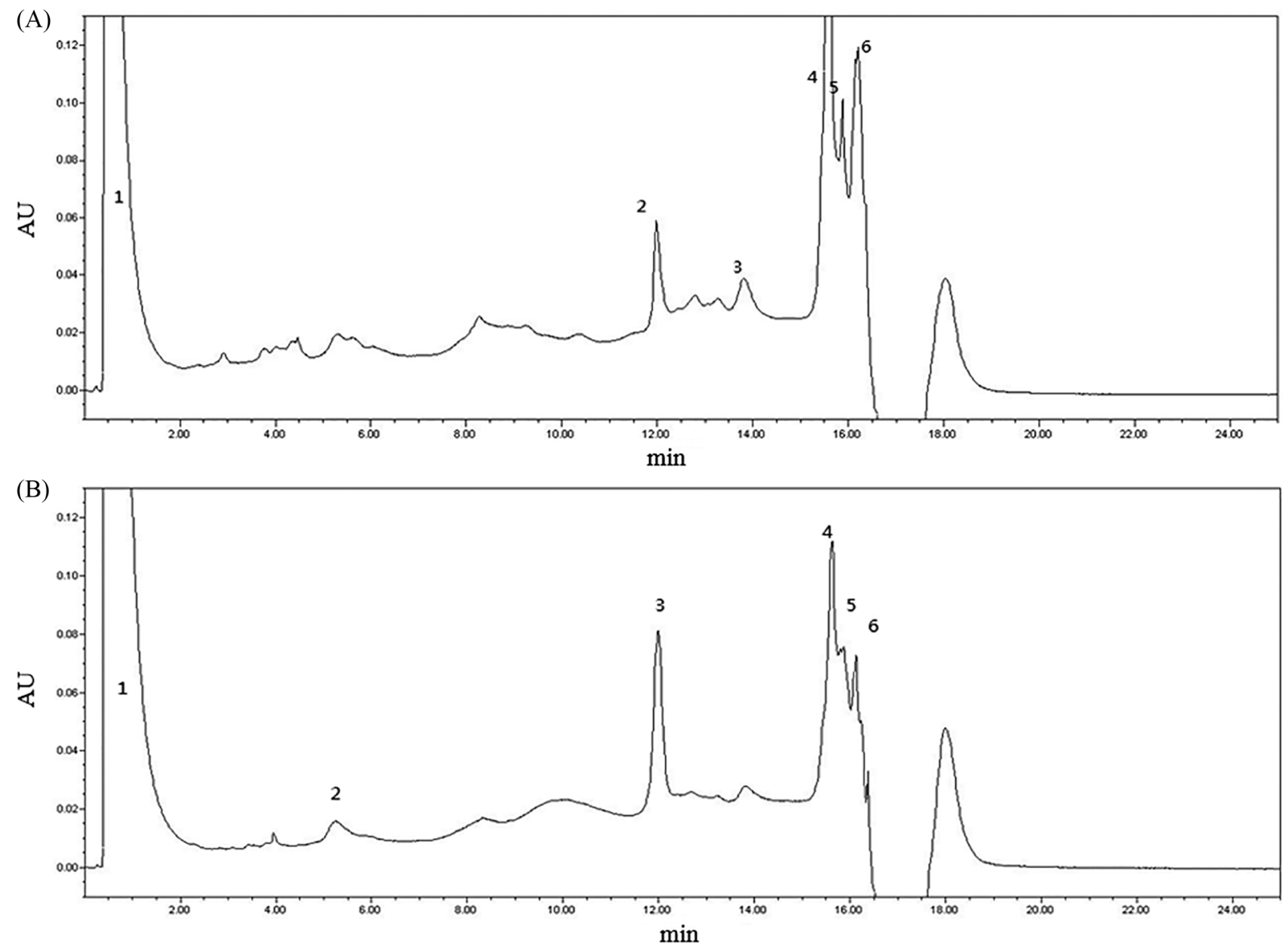

Fig. 1. Chromatograms of influenza H1N1 antigen and seasonal influenza monovalent H1N1 vaccine. RP-HPLC chromatograms of H1N1 antigens $(A)$ and H1N1 vaccine $(B)$ are represented. Fractions from RP-HPLC chromatograms were designated with numbers according to the eluted time. 

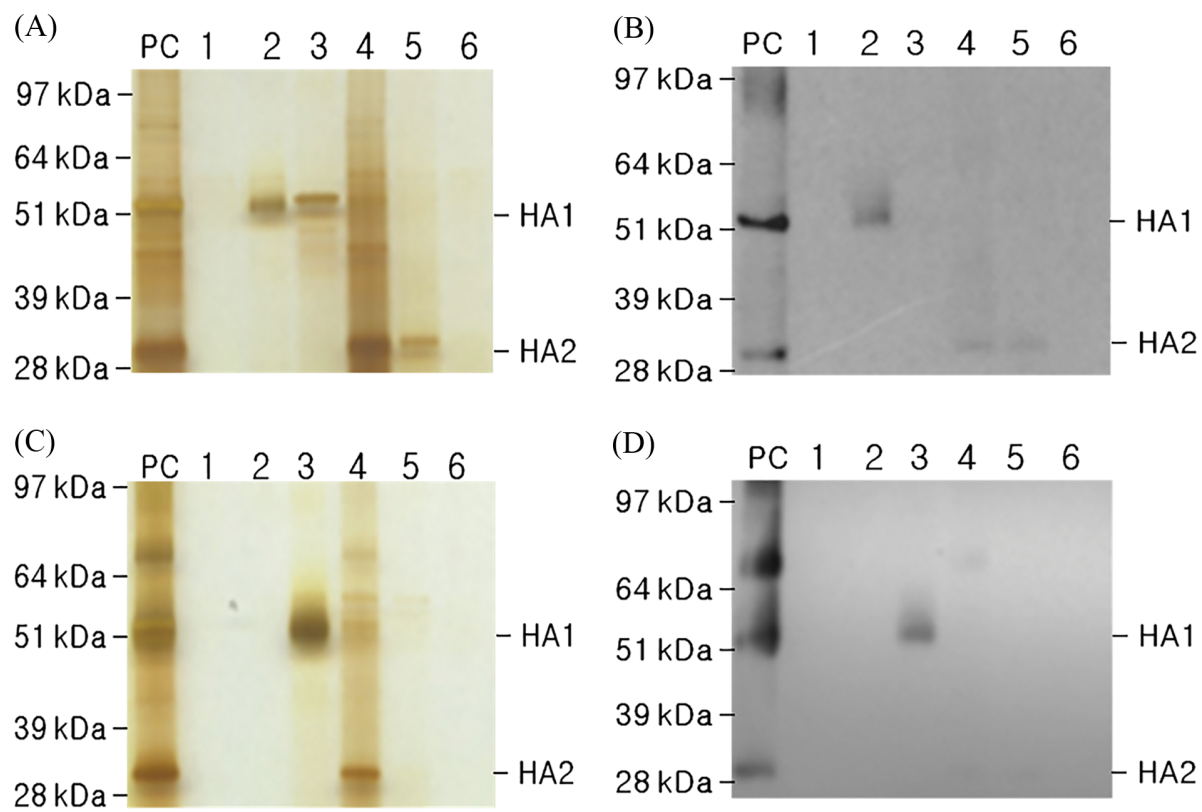

Fig. 2. Silver staining ( $A$ and $C$ ) and Western blot ( $B$ and $D)$ of RP-HPLC fractions from standard H1N1 antigen (A and $B)$ and seasonal influenza H1N1 vaccine (C and D). Elutes separated from RP-HPLC chromatograms were subjected to silver staining and Western blot. Specific H1N1 anti-HA serum was used to label HA protein.
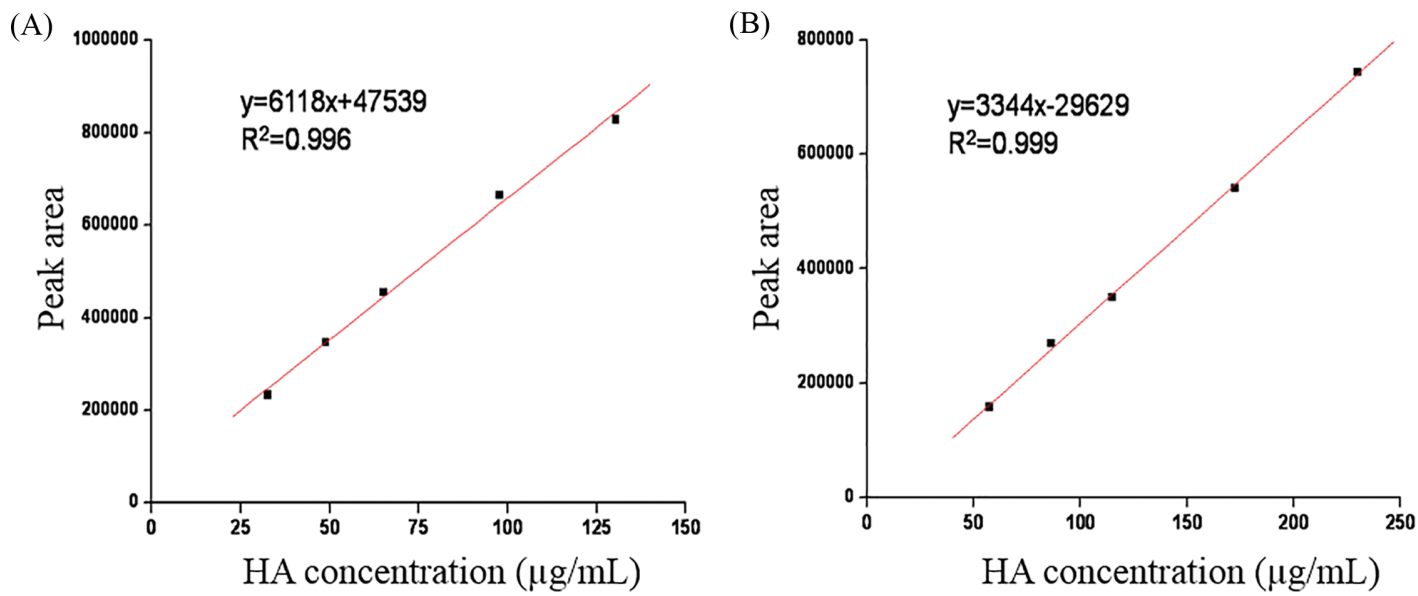

Fig. 3. Plots of calibration represent $H A 1$ peak areas from $H 1 N 1$ antigen (A) and seasonal influenza H1N1 vaccine (B). Different amounts of HA1 in a concentration-dependent manner were processed for RP-HPLC, and the peak area from 12 min was analyzed. The peak area from RP-HPLC was highly correlated with the concentration of HA1.

(Fig. 3A) and H1N1 vaccine (Fig. 3B), showing at least 0.99 of the coefficient of determination $\left(\mathrm{R}^{2}\right)$. The repeatability of the peak areas and retention times for H1N1 antigen and vaccine were also examined using four different samples. The relative standard deviations (RSD) calculated from the HA1 peak area and retention time were 1.03 $2.24 \%$ and $0.06 \sim 0.08 \%$, respectively, indicating superior repeatability (Table 1). Accuracy of HA content was determined by measurement of HA peak areas by two different people, and coefficient value $(\mathrm{CV})$ was in the $0.2 \sim 8.8 \%$ range between the two results (data not shown). The two
HA concentrations, with signal-to-noise ratios of $3: 1$ and $10: 1$, respectively, were defined as the LOD and LOQ, respectively (Table 2 ).

Comparison of HA content by RP-HPLC and SRID. To assess HA content $(\mu \mathrm{g} / \mathrm{mL})$ from $\mathrm{H} 1 \mathrm{~N} 1$ vaccines after RPHPLC, five HA1 concentrations of international standard antigens (NIBSC code: 09/294 and 11/202) were used as standards, and calibration curves were drawn based on the HA1 peak area. Correlation with HA content analyzed by SRID and RP-HPLC was evaluated from 12 lots of sea- 
Table 1. Repeatability of the HA1 peak area and retention time

\begin{tabular}{cccc}
\hline \hline & No. & Area & Retention time (min) \\
\hline & 1 & 207907 & 12.02 \\
& 2 & 209547 & 12.03 \\
H1N1 antigens & 3 & 204499 & 12.03 \\
& 4 & 208902 & 12.04 \\
& 5 & 205727 & 12.05 \\
& 6 & 209614 & 12.04 \\
& Average & 207316 & 12.03 \\
& RSD (\%) & 1.03 & 0.08 \\
\hline \multirow{4}{*}{ H1N1 vaccines } & 1 & 183050 & 12.05 \\
& 2 & 183960 & 12.05 \\
& 3 & 188573 & 12.05 \\
& 4 & 192404 & 12.04 \\
& 5 & 189547 & 12.03 \\
& 6 & 193271 & 12.05 \\
& Average & 188468 & 12.05 \\
& RSD (\%) & 2.24 & 0.06 \\
\hline
\end{tabular}

RSD: Relative Standard Deviation.

sonal H1N1 vaccines produced from 2013 to 2014 (Table 3). HA1 content from $10 \mathrm{H} 1 \mathrm{~N} 1$ vaccine samples were analyzed three times by RP-HPLC under the same conditions as the calibrants, and mean values of HA1 peak area were extrapolated from the calibration curves. Then, ratios of HA content (RP-HPLC/SRID) were designated as \%. The values of ratios were between 1.74 and 1.99 with an average of 1.85 , suggesting that the HA1 content analyzed by RPHPLC was highly correlated with the values of HA1 measured by SRID.

\section{DISCUSSION}

In 2009, an influenza pandemic swept throughout the world, resulting in massive social and economic costs. Fortunately, development of safe and effective vaccines saved many lives and dampened the pandemic. Based on past experiences, the most effective way to tackle an influenza
Table 3. HA content results obtained by SRID and RP-HPLC methods from seasonal influenza $\mathrm{H} 1 \mathrm{~N} 1$ vaccines

\begin{tabular}{cccc}
\hline \hline Lot No & $\begin{array}{c}\text { HA1 } \\
\text { by RP-HPLC }\end{array}$ & $\begin{array}{c}\text { HA1 } \\
\text { by SRID }\end{array}$ & RP-HPLC/SRID \\
\hline v121092 & $416 \pm 13.0$ & 223 & 1.87 \\
v121093 & $424.8 \pm 11.5$ & 239 & 1.78 \\
v121094 & $411.6 \pm 14.2$ & 229 & 1.80 \\
v121096 & $368.8 \pm 20.1$ & 206 & 1.79 \\
v121097 & $395.6 \pm 11.8$ & 215 & 1.84 \\
v121098 & $378.8 \pm 14.2$ & 207 & 1.83 \\
v121099 & $366.7 \pm 6.6$ & 211 & 1.74 \\
v121100 & $397.3 \pm 16.0$ & 211 & 1.88 \\
v121101 & $401.8 \pm 18.7$ & 224 & 1.79 \\
v121106 & $376.6 \pm 14.1$ & 195 & 1.93 \\
v121107 & $391.3 \pm 15.5$ & 202 & 1.94 \\
v121108 & $394.0 \pm 18.3$ & 198 & 1.99 \\
\hline Average & 393.6 & 213.3 & 1.85 \\
\hline
\end{tabular}

pandemic might be prevention through vaccination rather than focusing on treatment after an outbreak. For rapid development of vaccines in response to an influenza pandemic, it is critical to speed up clinical tests to determine the appropriate amount of antigen per vaccine dose. Quick measurement of the antigen contents of vaccines can facilitate clinical trials and early access to vaccines for the good of public health. However, the time required to produce SRID reagents is considered a primary drawback, resulting in delayed availability of vaccines during emergencies. During the last pandemic in 2009, Korea had to postpone vaccine inoculation even after the $\mathrm{H} 1 \mathrm{~N} 1$ influenza vaccine was developed due to a delay in securing standard substances from the WHO. In this study, H1N1 standard antigens and H1N1 vaccines produced by a domestic manufacturer were examined by RP-HPLC. The HA1 peak was isolated and its content was measured from the H1N1 antigen and vaccine. The silver staining and Western blot results revealed that the peak elutes from $\mathrm{H} 1 \mathrm{~N} 1$ antigen and vaccine were specific for HA1 protein. The content of HA1 from the

Table 2. Precision within laboratory

\begin{tabular}{cccccc}
\hline \hline Vaccine (lot) & Amount of HA $\left(1^{\text {st }}\right)$ & Amount of HA $\left(2^{\text {nd }}\right)$ & Amount of HA $\left(3^{\text {rd }}\right)$ & Average & RSD $(\%)$ \\
\hline v121092 & $426.5 \pm 3.6$ & $400.7 \pm 4.3$ & $422.58 \pm 8.2$ & $416.6 \pm 13.0$ & 3.1 \\
v121093 & $439.5 \pm 1.8$ & $415.3 \pm 2.2$ & $419.64 \pm 5.3$ & $424.8 \pm 11.5$ & 2.7 \\
v121094 & $425.4 \pm 1.9$ & $393.8 \pm 2.9$ & $415.48 \pm 5.8$ & $411.6 \pm 14.2$ & 3.4 \\
v121096 & $401.6 \pm 6.9$ & $360.9 \pm 7.8$ & $397.86 \pm 2.1$ & $368.8 \pm 20.1$ & 5.2 \\
v121097 & $407.1 \pm 4.7$ & $381.1 \pm 2.1$ & $398.54 \pm 3.6$ & $395.6 \pm 11.8$ & 2.9 \\
v121098 & $394.1 \pm 1.4$ & $361.6 \pm 2.2$ & $380.82 \pm 4.5$ & $378.8 \pm 14.2$ & 3.7 \\
v121099 & $374.2 \pm 2.2$ & $363.8 \pm 3.6$ & $362.13 \pm 4.9$ & $366.7 \pm 6.6$ & 1.8 \\
v121100 & $396.6 \pm 3.0$ & $379.3 \pm 4.7$ & $415.95 \pm 2.8$ & $397.3 \pm 16.0$ & 4.0 \\
v121101 & $403.0 \pm 1.6$ & $379.5 \pm 2.4$ & $422.95 \pm 4.5$ & $401.8 \pm 18.7$ & 4.6 \\
v121106 & $379.7 \pm 3.8$ & $359.4 \pm 3.8$ & $390.68 \pm 4.7$ & $376.6 \pm 14.1$ & 3.7 \\
v121107 & $394.7 \pm 3.3$ & $372.1 \pm 4.1$ & $407.09 \pm 4.2$ & $391.3 \pm 15.5$ & 3.9 \\
v121108 & $396.0 \pm 6.3$ & $372.2 \pm 3.9$ & $413.79 \pm 2.9$ & $394.0 \pm 18.3$ & 4.6 \\
\hline
\end{tabular}

RSD: Relative Standard Deviation. 
peak area was highly conserved, with a retention time of 12 minutes. In the next experiment, the content of HA1 derived from RP-HPLC was compared with that from SRID. The results showed a relatively good correlation of HA content between RP-HPLC and SRID, suggesting that determination of HA1 content by RP-HPLC is accurate compared with SRID. However, based on our results, the contents of HA from RP-HPLC were 1.85-fold higher compared to SRID. This discrepancy in HA contents between the methods may be due to differences in how HA concentration is represented between SRID and RP-HPLC. HA1 analyzed by SRID represents the amount of HA1 that reacts only with sheep polyclonal anti-HA antibody, whereas HA1 analyzed by RP-HPLC represents the total amount of denatured HA1.

In conclusion, based on the results of this study, the HA contents of manufacturing produced influenza vaccines can be measured by the RP-HPLC method, which will help to expedite vaccine production in urgent cases as a replacement for SRID.

\section{ACKNOWLEDGMENTS}

This research was supported by a grant (11171MFDS365) from Ministry of Food and Drug Safety in 2011. We would like to thank Green Cross Corp. for providing vaccines.

\section{REFERENCES}

1. Kitler, M.E., Gavinio, P. and Lavanchy, D. (2002) Influenza and the work of the World Health Organization. Vaccine, 20 Suppl 2, S5-S14.

2. Wood, J.M., Schild, G.C., Newman, R.W. and Seagroatt, V.
(1977) An improved single-radial-immunodiffusion technique for the assay of influenza haemagglutinin antigen: application for potency determinations of inactivated whole virus and subunit vaccines. J. Biol. Stand., 5, 237-247.

3. Willkommen, H., Platen, S. and Staber, H. (1983) The influence of $\mathrm{pH}$ and ionic strength on the single radial immunodiffusion test in qualitative assay of influenza virus haemagglutinin. Acta Virol., 27, 407-411.

4. Gerdil, C. (2003) The annual production cycle for influenza vaccine. Vaccine, 21, 1776-1779.

5. Gerdil, C. (2003) Using the strains and getting the vaccine licensed--a vaccine manufacturer's view. Dev. Biol. (Basel), 115, 17-21.

6. Kapteyn, J.C., Saidi, M.D., Dijkstra, R., Kars, C., Tjon, J.C., Weverling, G.J., de Vocht, M.L., Kompier, R., van Montfort, B.A., Guichoux, J.Y., Goudsmit, J. and Lagerwerf, F.M. (2006) Haemagglutinin quantification and identification of influenza A\&B strains propagated in PER.C6 cells: a novel RP-HPLC method. Vaccine, 24, 3137-3144.

7. Kapteyn, J.C., Porre, A.M., de Rond, E.J., Hessels, W.B., Tijms, M.A., Kessen, H., Slotboom, A.M., Oerlemans, M.A., Smit, D., van der Linden, J., Schoen, P. and Thus, J.L. (2009) HPLC-based quantification of haemagglutinin in the production of egg- and MDCK cell-derived influenza virus seasonal and pandemic vaccines. Vaccine, 27, 1468-1477.

8. Lorbetskie, B., Wang, J., Gravel, C., Allen, C., Walsh, M., Rinfret, A., Li, X. and Girard, M. (2011) Optimization and qualification of a quantitative reversed-phase HPLC method for hemagglutinin in influenza preparations and its comparative evaluation with biochemical assays. Vaccine, 29, 33773389.

9. Wood, J.M., Mumford, J., Schild, G.C., Webster, R.G. and Nicholson, K.G. (1986) Single-radial-immunodiffusion potency tests of inactivated influenza vaccines for use in man and animals. Dev. Biol. Stand., 64, 169-177. 